\title{
PS-007 NEGLECTED INFECTIOUS DISEASES
}

Nathalie Strub-Wourgaft. DNDi, Switzernad

10.1136/bmjgh-2016-000260.7

Achievement of the UN's Sustainable Development Goals (SDGs) will only be possible if the burden of neglected tropical diseases (NTDs) is significantly reduced. NTDs have an impact on population health, and are a drain on community resources, hindering economic development. The burden is particularly high in Africa, and urgent action is required on NTDs to enable the attainment of the SDGs.

The World Health Organization has listed 17 NTDs that impact one billion people worldwide and produced overwhelming evidence to show that their impact may be effectively controlled and, in many cases, eliminated or eradicated. The WHO Strategic and Technical Advisory Group for NTDs and partners adopted a roadmap for control, elimination and eradication which set targets for 2012-2020.

In its systematic assessment of the drug and vaccine landscape for neglected diseases up to 2011, DNDi found a persistent insufficiency in drug and vaccine development, and that new therapeutic products urgently need to be developed and delivered to improve control and potentially achieve elimination. It is significant that the second EDCTP programme includes neglected infectious diseases and more trial phases. DNDis focusses on the needs of neglected patients, and is developing treatments for sleeping sickness, leishmaniasis, Chagas disease, filariasis, HCV, paediatric HIV, and Mycetoma. In Africa there is an acute need for treatments for visceral leishmaniasis (VL), HIV-VL coinfection, post kalaazar dermal leishmaniasis, and cutaneous leishmaniasis. Mycetoma was recognized as an NTD by the WHA in 2016; this devastating disease affects remote populations; is poorly understood and lacks effective treatment. In Africa, 115 million people are at risk of onchocerciasis and 410 million people require preventive chemotherapy for lymphatic filariasis; mass administration programs are hampered by drugs that only kill the juvenile form of the worms, leaving only temporarily sterilized adults, requiring repeated administration over a period of decades. 